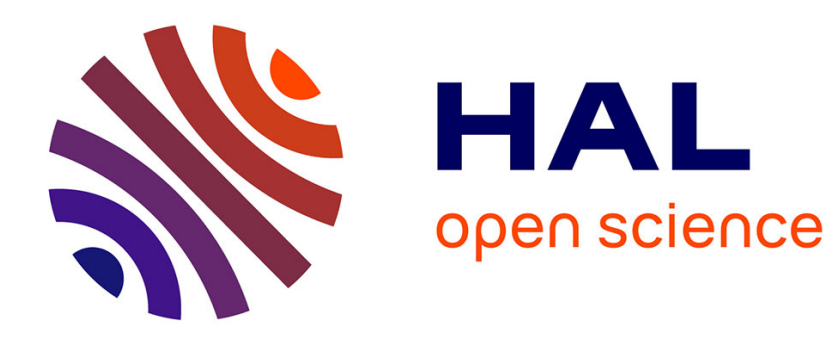

\title{
Un système de charge pour le VIVITRON
}

J.M. Helleboid

\section{- To cite this version:}

J.M. Helleboid. Un système de charge pour le VIVITRON. Revue de Physique Appliquée, 1988, 23

(9), pp.1457-1465. 10.1051/rphysap:019880023090145700 . jpa-00245971

\section{HAL Id: jpa-00245971 \\ https://hal.science/jpa-00245971}

Submitted on 1 Jan 1988

HAL is a multi-disciplinary open access archive for the deposit and dissemination of scientific research documents, whether they are published or not. The documents may come from teaching and research institutions in France or abroad, or from public or private research centers.
L'archive ouverte pluridisciplinaire HAL, est destinée au dépôt et à la diffusion de documents scientifiques de niveau recherche, publiés ou non, émanant des établissements d'enseignement et de recherche français ou étrangers, des laboratoires publics ou privés. 


\title{
Un système de charge pour le VIVITRON
}

\author{
J. M. Helleboid \\ Centre de Recherches Nucléaires, BP 2067037 Strasbourg Cedex, France
}

(Reçu le 15 octobre 1987, accepté le 17 mai 1988)

Résumé. - Un système de charge à courroie de type Van de Graaff a été choisi pour le Vivitron. Ainsi, classique dans son principe, il inclut néanmoins nombre de particularités qui sont discutées en détail. Les principales caractéristiques électrostatiques et mécaniques du système sont présentées, ainsi que l'état d'avancement du projet.

Abstract. - A Van de Graaff belt charging system has been chosen for the Vivitron. Although classical in its principle and conservative in its design, it includes different new features that will be discussed in detail. The main electrical and mechanical characteristics are also reviewed together with the status of the project.

\section{Introduction.}

Le Vivitron [1] (Fig. 1), en construction au C.R.N., est un accélérateur électrostatique de $35 \mathrm{MV}$ pour lequel un système de charge est en cours de développement [2-4].

La conception de principe nous a conduit au choix d'un système à courroie de type Van de Graaff intégrant nombre d'idées nouvelles, certaines fonda- mentales, qui en font un ensemble original et spécifique, bien adapté au cahier des charges du Vivitron.

Les principales caractéristiques électriques et mécaniques ont été calculées en tenant compte des contraintes imposées pour les autres aspects du projet, de manière à obtenir les performances souhaitées.

Les études de réalisation sont en cours.

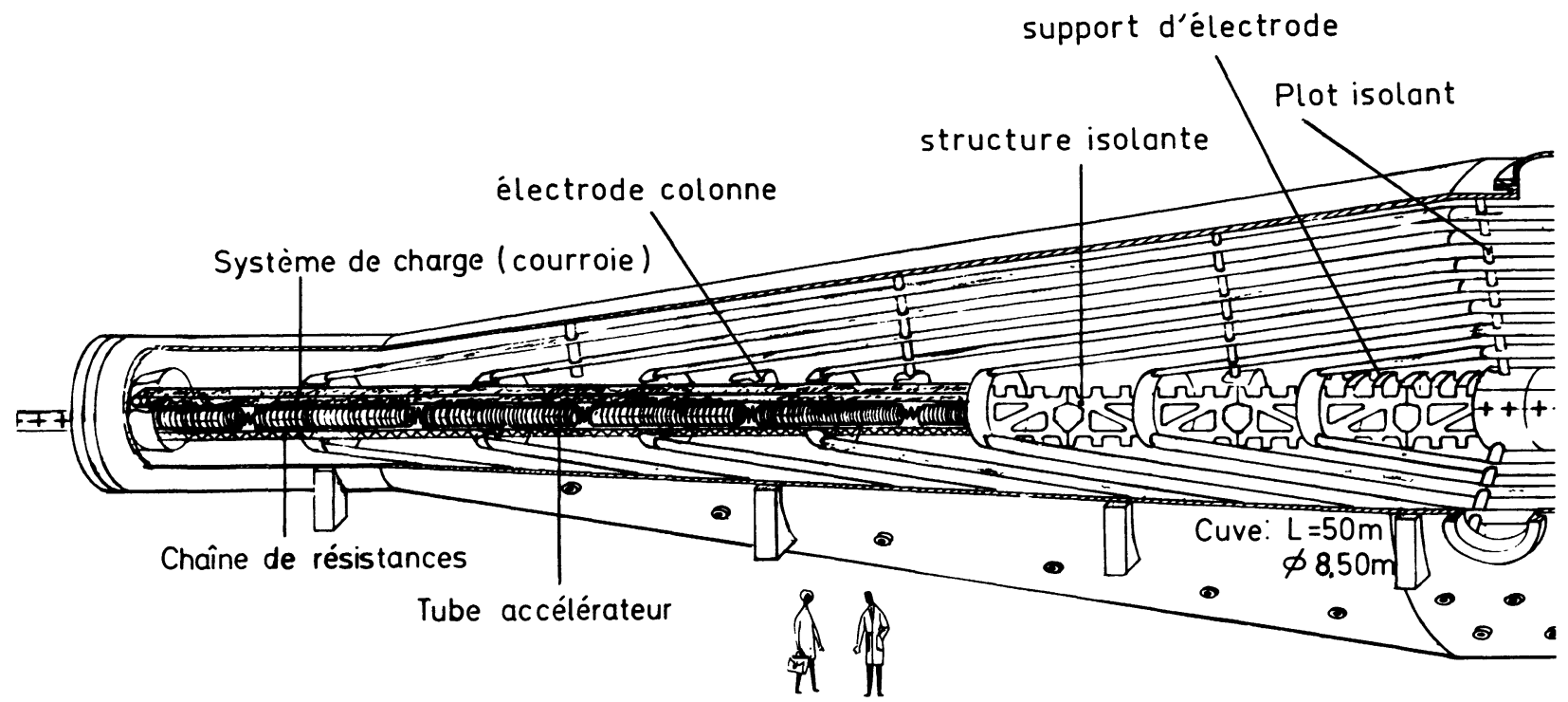

Fig. 1. - Le Vivitron : un accélérateur électrostatique de $35 \mathrm{MV}$.

[The Vivitron : a $35 \mathrm{MV}$ electrostatic accelerator.] 


\section{Objectif.}

Le système de charge du Vivitron doit délivrer un courant de $500 \mu \mathrm{A}$ pour une haute tension jusqu'à $35 \mathrm{MV}$ avec une stabilité comparable (ou meilleure) à celle des grands tandems Van de Graaff. Cette valeur d'intensité a été déduite par extrapolation d'une valeur courante de $320 \mu \mathrm{A}$ à $18 \mathrm{MV}$ pour l'actuel tandem MP en fonctionnement au C.R.N.

\section{Contraintes.}

Le système de charge doit être implanté dans un espace transversalement limité, tant du point de vue géométrique qu'électrique, entre le tube accélérateur et les électrodes-colonne, leurs supports, et la structure isolante longitudinale (Figs. 1 et 2).

Situé à l'intérieur de la «colonne » il doit comporter un minimum de parties métalliques pour respec- ter la philosophie générale de construction du Vivitron, en évitant de focaliser les lignes de champ et donc de canaliser les décharges vers l'intérieur. De fait, et compte tenu de la présence du champ électrique longitudinal d'accélération $\bar{E} \simeq$ $18 \mathrm{kV} / \mathrm{cm}$, les fixations, supports ou guidages éventuels doivent se situer dans les sections mortes, soit sur environ $220 \mathrm{~mm}$ au plus tous les $2,82 \mathrm{~m}$ (Fig. 1). Les distances le long de l'axe, depuis l'électrode isolée jusqu'à la masse, sont d'environ $25 \mathrm{~m}$.

L'ensemble est sous atmosphère d'hexafluorure de soufre $\left(\mathrm{SF}_{6}\right)$ à une pression nominale de 8 bars absolus (valeur maximale 12 bars).

\section{Choix.}

Bien que de nombreux principes de charge existent, ou puissent être imaginés, il a été jugé raisonnable

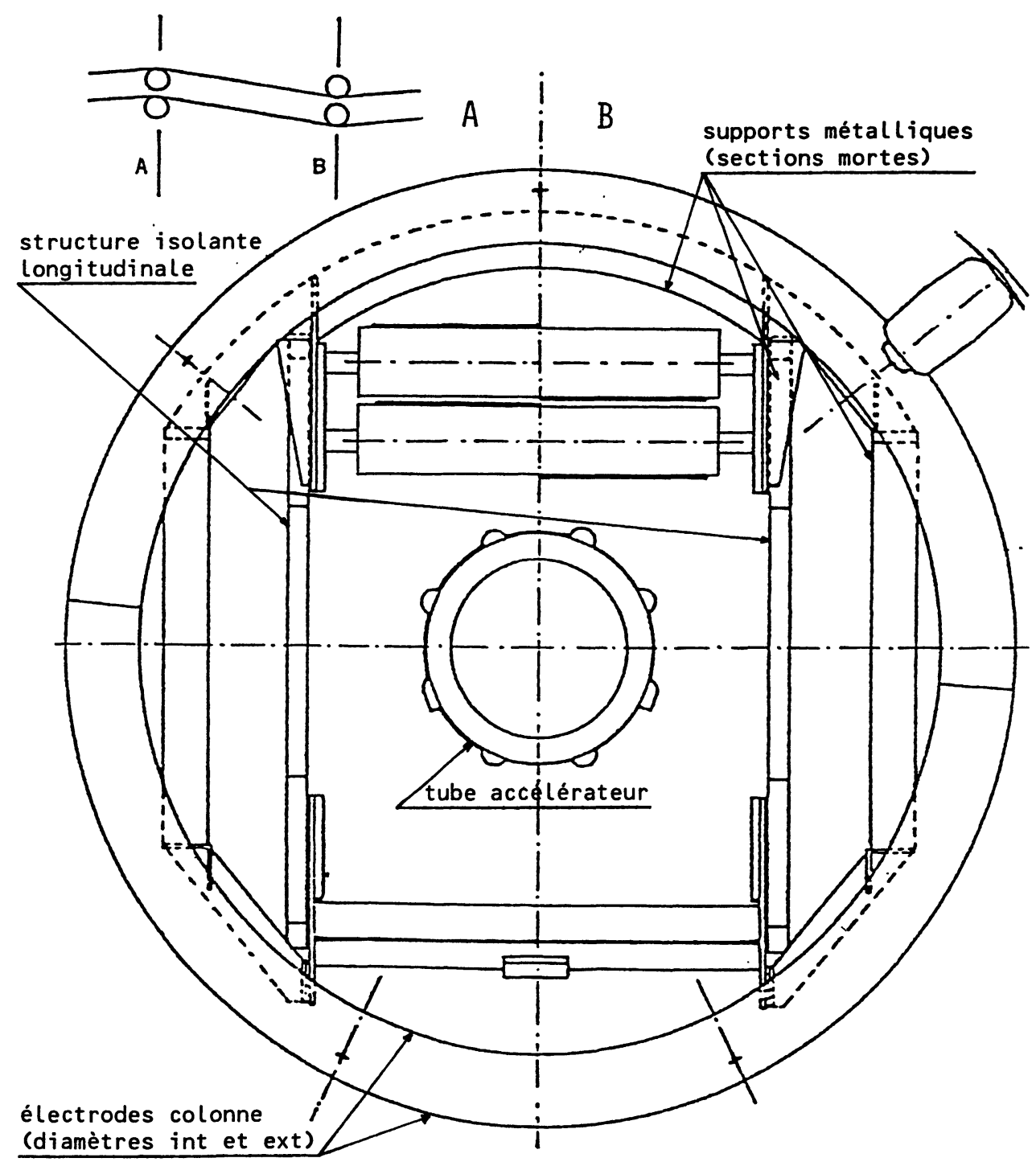

Fig. 2. - Section droite de la colonne du Vivitron montrant la position de la courroie dans deux sections mortes successives : A (gauche) et $\mathrm{B}$ (droite).

[Column cross-section and belt location in two successive dead-sections : A (left) and B (right).] 
de s'en tenir aux systèmes classiques pour de grands accélérateurs tandem. Des systèmes à chaîne (de type pelletron ou laddertron) ou à courroie, le dernier type a été retenu pour diverses raisons parmi lesquelles:

- la grande expérience acquise avec le système à courroie de l'accélérateur MP du C.R.N. et son bon fonctionnement pour une intensité nominale de $320 \mu \mathrm{A}$ à haute tension maximale de $18 \mathrm{MV}$;

- la possibilité d'intégrer dans la conception d'un tel système de nouvelles caractéristiques, issues d'idées originales [2, 3], qui nous semblent devoir apporter une amélioration des performances actuelles (contrainte électrique moindre, niveau de charges parasites diminué...) et donc de la fiabilité ;

- la modeste intensité requise pour le Vivitron : $I=500 \mu \mathrm{A}$, comparée avec les $700 \mu \mathrm{A}(2 \times 350)$ qui peuvent être obtenus sur le tandem MP à $15 \mathrm{MV}$;

- la récente disponibilité, auprès d'une firme suédoise, d'un nouveau type de courroie, à prix raisonnable pour de grandes dimensions, et déjà expérimentée sur plusieurs accélérateurs [5-7].

\section{Conception de principe.}

Dans tous les systèmes de charge actuels l'intensité maximale est toujours très inférieure à la valeur limite qui peut être calculée, à partir des lois de l'électrostatique, pour un système idéal. Par rapport à ce dernier de nombreux facteurs de limitation interviennent en effet. Ils diminuent aussi la fiabilité pour des performances données.

La conception du système de charge pour le Vivitron propose des solutions originales qui tendent à réduire ces limitations tout en augmentant la fiabilité.

5.1 Courroie PASSANTE. - Plutôt qu'un système de charge n'occupant, comme dans les tandems actuels, qu'un seul côté de la machine, entre masse et électrode isolée, il a été décidé d'utiliser une courroie passante, c'est-à-dire tendue d'une extrémité à l'autre de la machine, étendant ainsi au système de charge la symétrie tandem de la colonne et du tube accélérateur (Fig. 3). La courroie, de $\sim 100 \mathrm{~m}$ de longueur et $520 \mathrm{~mm}$ de largeur, peut alors être électriquement divisée en quatre portions actives, transportant successivement et alternativement une densité de charge égale et opposée entre la masse et l'électrode isolée, soit le double des systèmes existants.

Pour un même courant total transporté la densité de charge sur la courroie est alors divisée par deux comme l'est la contrainte électrique transversale qui en résulte.

De plus, la tension de courroie à partir des extrémités de la cuve, évite l'application d'une contrainte mécanique longitudinale sur la colonne.

5.2 Structure déCOUPLÉE. - Les brins de courroie en regard, séparés par une distance $d$ (Fig. 4), portent des charges égales et opposées. En l'absence de tout conducteur interne, placé entre les brins, et pour un système supposé plan et infini où les conducteurs extérieurs, entourant la courroie, seraient distants de $D$, le champ électrique à l'extérieur des brins $E_{\text {ext }}=\sigma / \varepsilon_{0} \times d / D$ est limité par $d / D$. Pour une densité de charge $\sigma$ donnée, il est d'autant plus faible que $d<D$, soit, à encombrement constant ( $D$ donné), que les brins sont plus proches. C'est l'effet de découplage; le système de charge et le reste de l'accélérateur fonctionnent quasi-indépendamment, ce qui permet d'adapter la géométrie des conducteurs extérieurs aux nécessités de la tenue électrique longitudinale, presque sans tenir compte de la présence du système de charge. La comparaison peut être faite entre la figure 5 qui représente la situation dans le MP et la figure 6 pour une structure identique mais où les brins sont rapprochés. Dans ce dernier cas, la distorsion du champ, résultant de la discontinuité des conducteurs extérieurs (barres de gradient dans cet exemple), n'est plus une cause de limitation comme pour le cas précédent.

Il semble qu'il soit alors possible de supprimer

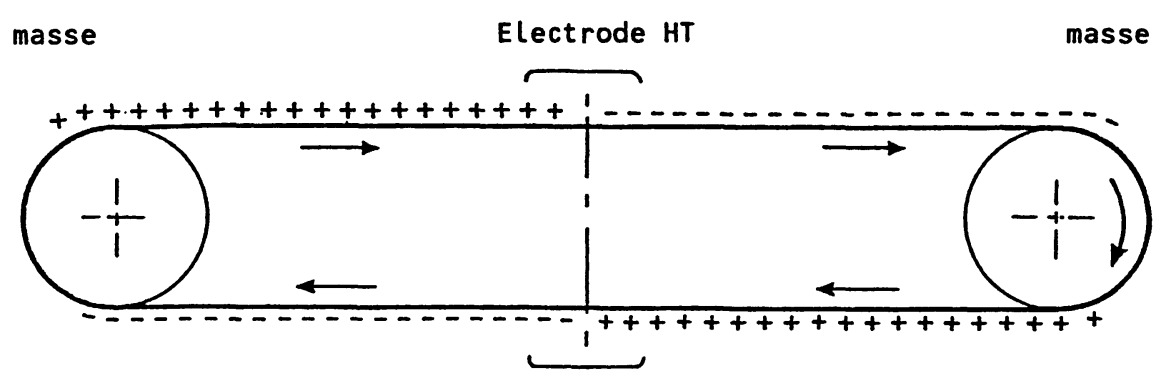

Fig. 3. - Courroie passante (symétrie tandem) présentant 4 portions chargées successivement en + et en - défilant respectivement de la masse vers l'électrode isolée et inversement.

[Symmetrical belt arrangement with four oppositely charged portions, each running from ground to the terminal and then back to ground.] 

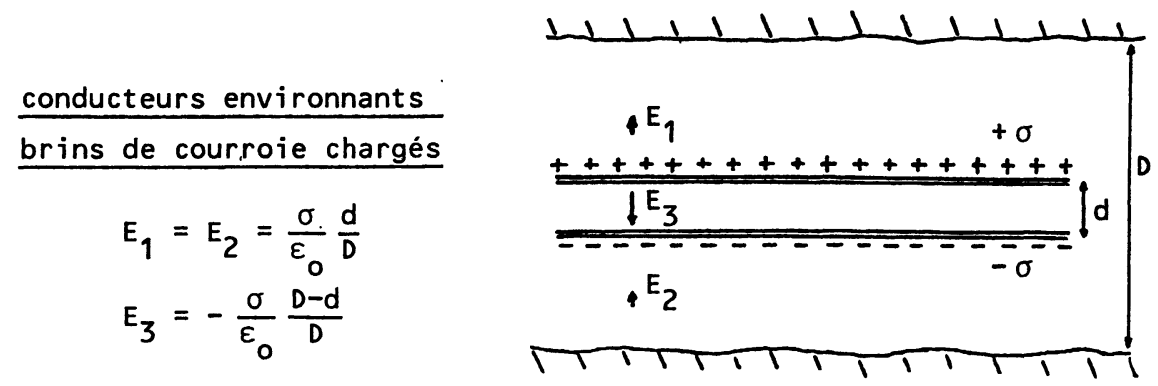

Fig. 4. - Principe de la structure découplée : le champ externe $E_{1}=E_{2}$ est d'autant plus faible que $d \ll D$.

[Principle of the decoupled structure : Two facing belt portions, equally but oppositely charged, induce quasi-uniform fields. The farer the nearby conductors $(D)$ compared with belt portions distance $(d)$, the higher the internal field $E_{3}\left(\max\right.$ value $\left.=\frac{\sigma}{\varepsilon_{0}}\right)$, the lower the external field $E_{1}=E_{2}$.]

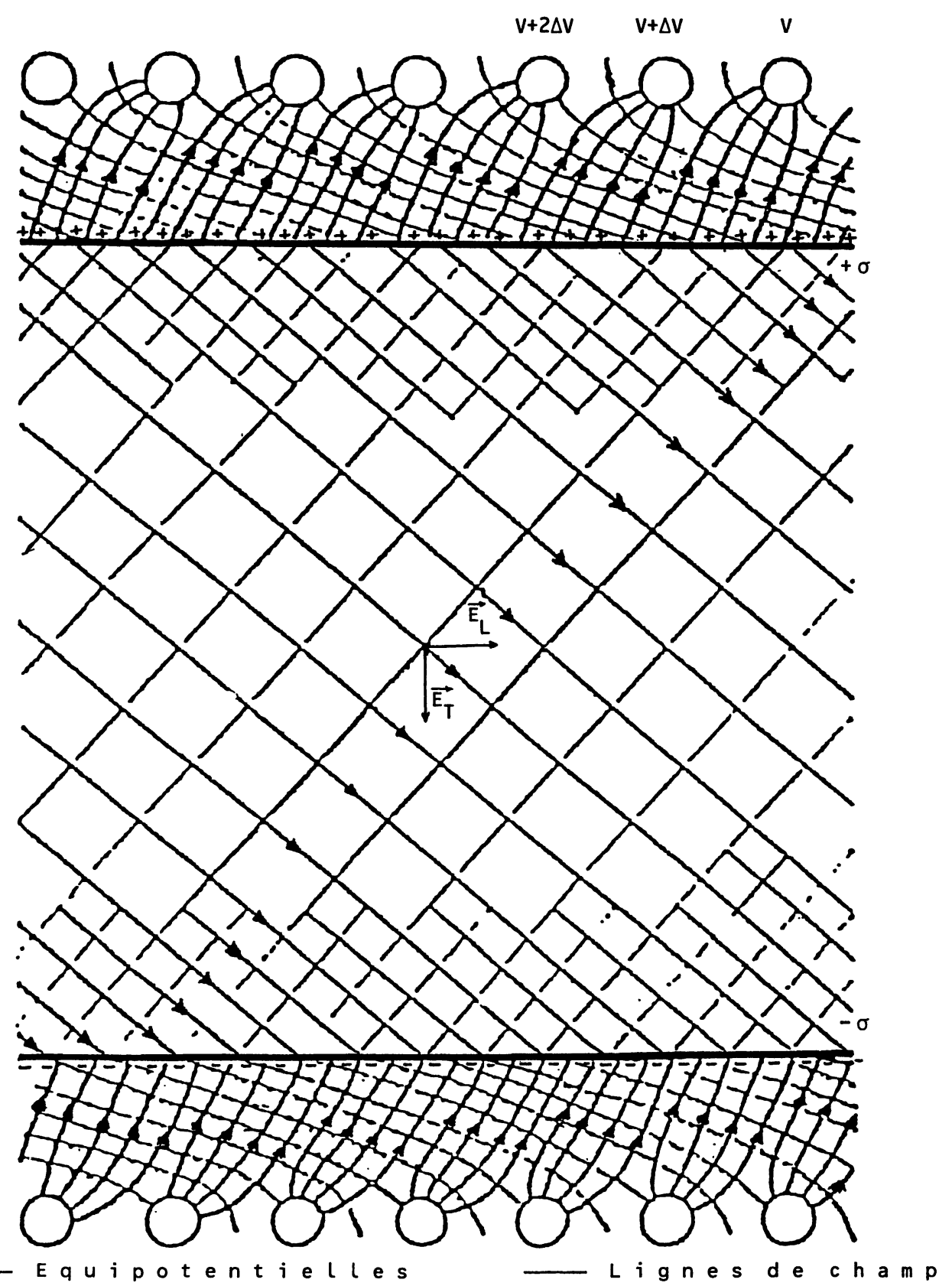

Fig. 5. - Distribution du potentiel et du champ électrique pour une structure semi-ouverte; exemple du MP.

[Potential and field lines for a semi-open structure. Example of the MP.] 


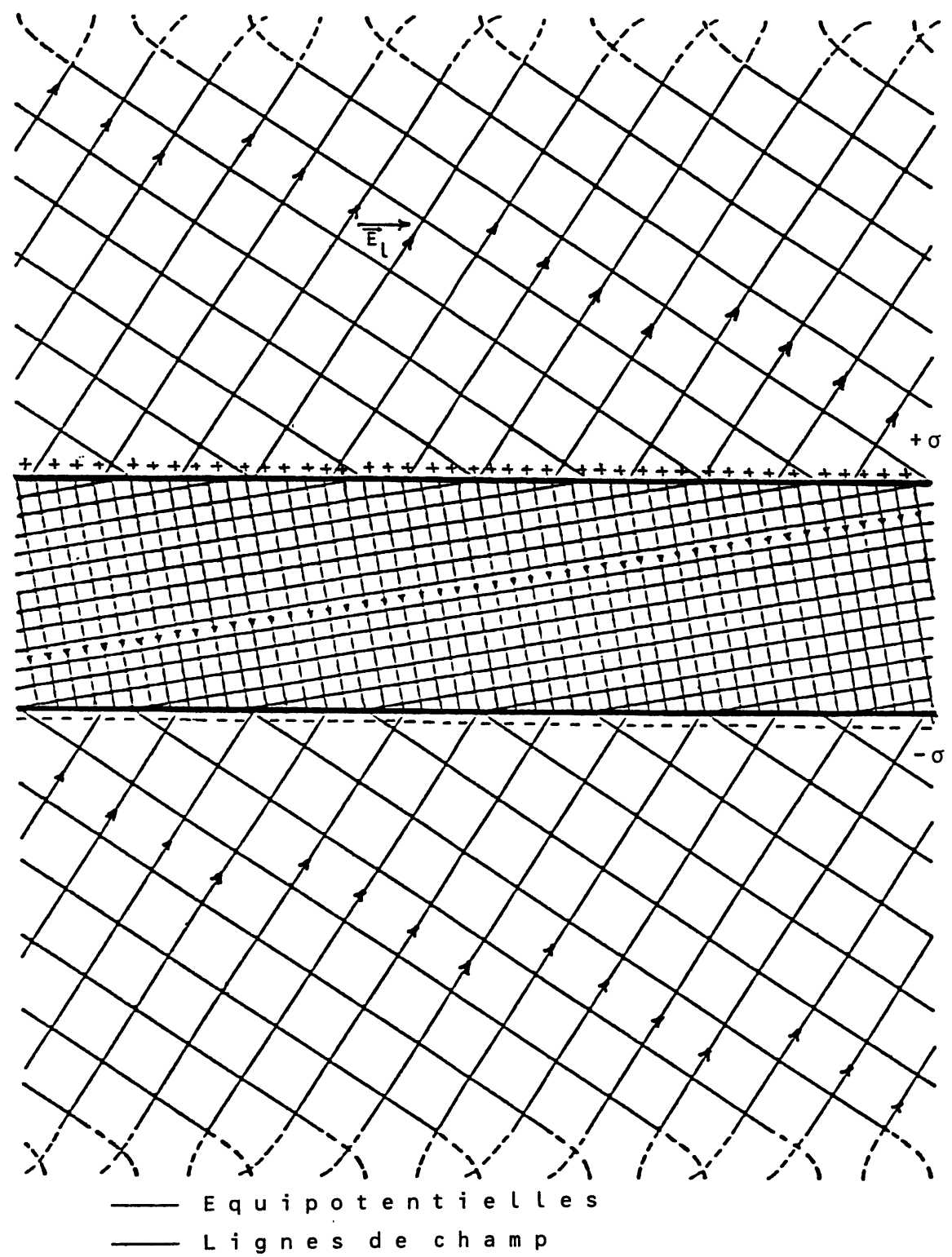

Fig. 6. - Distribution du potentiel et du champ électrique pour une structure découplée de même encombrement que pour le MP (Fig. 5).

[Potential and field lines for a decoupled structure arrangement with the same overall dimensions that for the MP. Nearby conductors (not drawn) are located horizontally in the upper and lower parts of the figure.]

également les barres de gradient externes, renforçant encore l'effet de découplage, en augmentant la distance $D$, et évitant la présence de pièces conductrices à l'intérieur de la colonne.

\subsection{DÉPÔT CONTRÔLÉ ET ÉQUILIBRE DES CHARGES} Comme à l'ordinaire c'est la face externe de la courroie qui porte les charges. En structure découplée, la limitation est directement liée, non seulement à la valeur des densités de charge, mais à leur assymétrie. Aussi un système particulier de commutation a-t-il été conçu (Fig. 7) qui limite le nombre d'ioniseurs et particulièrement de «collecteurs » dont l'efficacité est mal contrôlée. Ainsi trois des quatre commutations $(+\sigma \rightarrow-\sigma$ et inversement) sont assurés chacune par un seul ioniseur jouant un double rôle de collection $(\mp \sigma \rightarrow o$ ) et de charge $(o \rightarrow \pm \sigma)$, un peu comme dans les machines Felici, mais régulé en courant pour contrôler le transfert de charge. La dernière commutation est assurée, comme usuellement, par un éliminateur (ioniseur à la masse, $\pm \sigma \rightarrow o \pm \Delta \sigma$ ) suivi d'un ioniseur de charge $(o \rightarrow \mp \sigma)$ régulé en courant.

5.4 VITESSE RÉDUITE. - Grâce aux dispositions précédemment décrites la vitesse de la courroie peut être réduite tout en conservant une contrainte électrique plus faible que dans les systèmes classiques (Fig. 8). Cela diminue notablement la puissance perdue par frottements gazeux $\left(\propto v^{3}\right)$ et ainsi la 


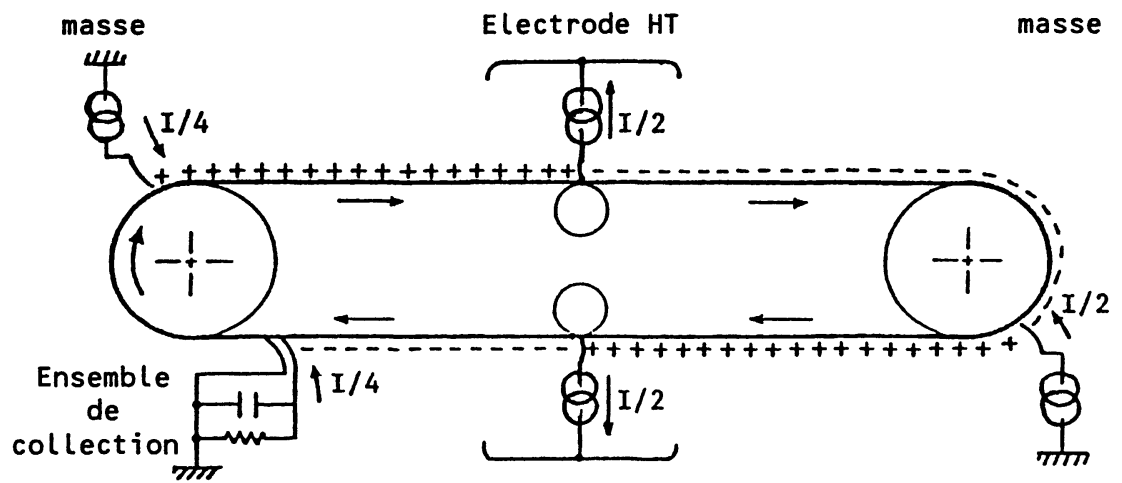

Fig. 7. - Système de commutation des charges pour le Vivitron.

[Charge commutation system for the Vivitron for a total current $I$.]

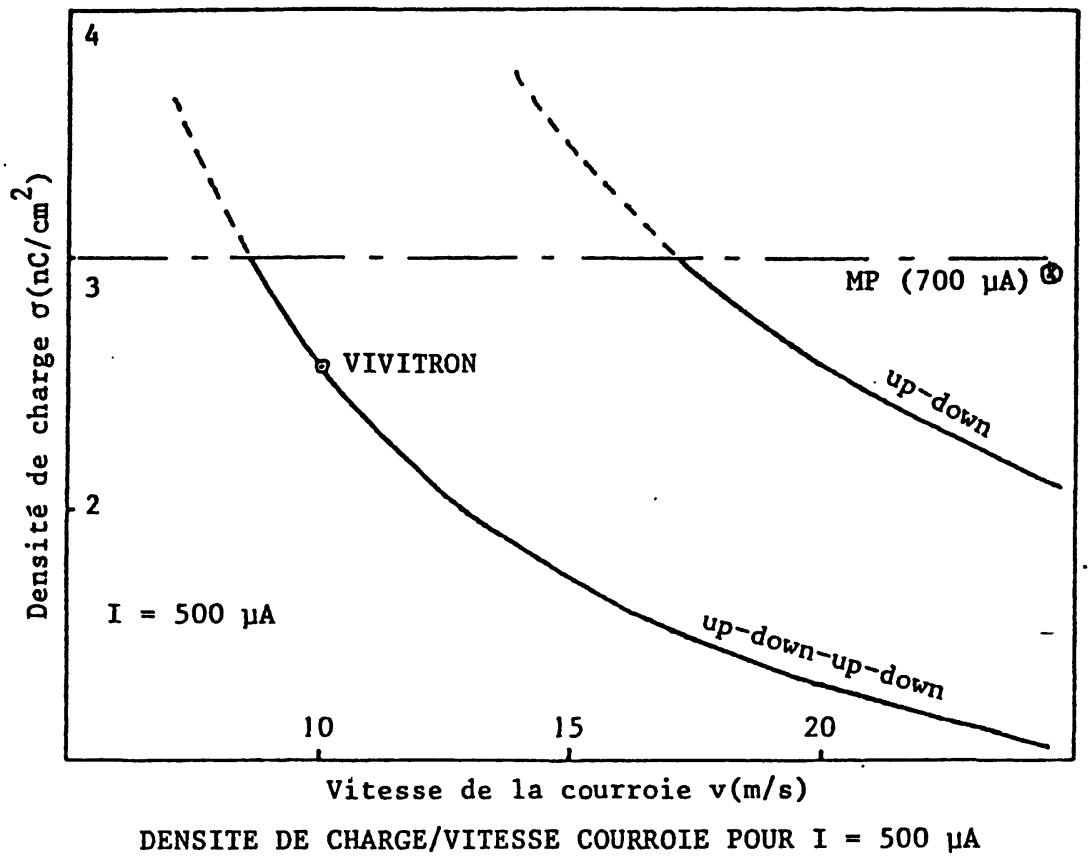

Fig. 8. - Densité de charge $\sigma$ en fonction de la vitesse de défilement pour un courant total transporté $I=500 \mu \mathrm{A}-$ cas du MP et du Vivitron.

[Charge density $\sigma$ versus belt speed for $I=500 \mu \mathrm{A}$ for the cases of the MP and of the Vivitron.]

production de charges parasites d'origine triboélectrique.

5.5 SUPPORTS. - Des rouleaux peuvent être utilisés, mais la sustentation par coussin de gaz $\mathrm{SF}_{6}$ est envisagée pour réduire les frottements et éviter les problèmes de roulements (Fig. 9).

5.6 TYPE DE COURROIE. - Un nouveau type de courroie (Fig. 10), déjà partiellement expérimenté dans des accélérateurs [5-7] et probablement satisfaisant dans les conditions Vivitron, est actuellement sur le marché, palliant la quasi impossibilité d'obtenir une courroie classique de $100 \mathrm{~m}$ dont le prix serait de plus prohibitif.

\section{Principales caractéristiques.}

Le tandem MP fonctionnant aisément avec une densité de charge $\sigma$ de $3 \mathrm{nC} / \mathrm{cm}^{2}$, la vitesse de la courroie du Vivitron a été fixée à $10 \mathrm{~m} / \mathrm{s}(24,5 \mathrm{~m} / \mathrm{s}$ dans le MP) pour laquelle $\sigma$ n'est que de $2,6 \mathrm{nC} / \mathrm{cm}^{2}$ pour $I=500 \mu \mathrm{A}$ (Fig. 9).

Les commutations seront assurées au début par des peignes classiques mais plus fins $(\varnothing \sim 0,1 \mathrm{~mm}$, 120 mesh).

La puissance nominale est de $25 \mathrm{~kW}$, incluant $V \times I=17,5 \mathrm{~kW}$ à $35 \mathrm{MV}$ et $500 \mu \mathrm{A}(6 \mathrm{~kW}$ pour le MP à $18 \mathrm{MV})$ et la friction sur le gaz $\sim 3 \mathrm{~kW}(14 \mathrm{~kW}$ pour le MP).

La courroie de $520 \mathrm{~mm}$ de large et $100 \mathrm{~m}$ de long 


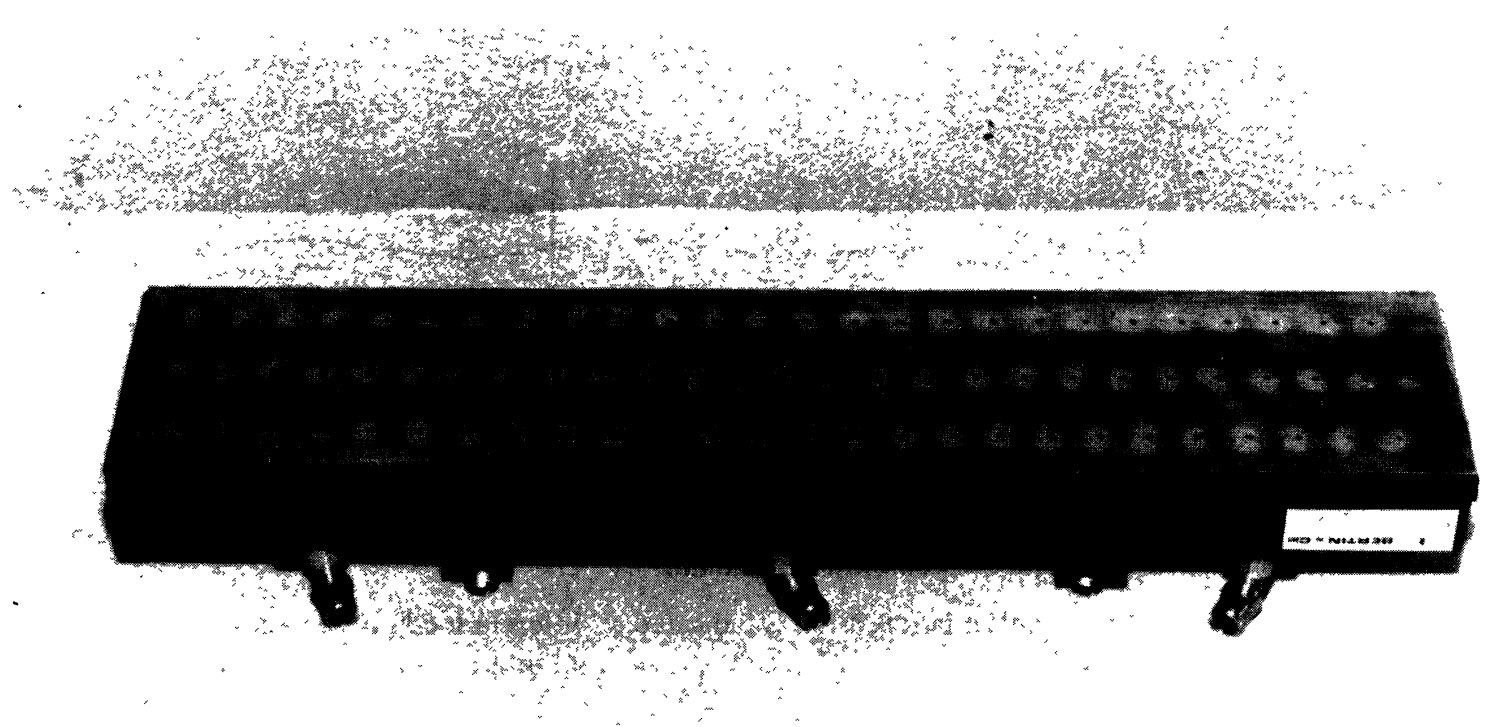

Fig. 9. - Prototype d'un support à coussin de gaz pour la courroie du Vivitron.

[Prototype of a gas blower support for the belt.]

\section{Tekniska Data}

Refererande till dagens telefonsamtal översänder vi tekniska data för transportband typ WE 16/2 0+10 Nitril och WTO 25.

Typbeteckning

Antal inlägg

Vävkvalitet

Ytbeläggning

Täckplattor

Brotthållfasthet

per $\mathrm{cm}$ bandbredd

Töjning vid

Törsp./arbetsbel.

Total tjocklek

Minsta rull- $\varnothing$

Arbetstemperatur

Max. bandbredd

Friktionskoefficient (kromstål)

$\begin{array}{cl}\begin{array}{c}\text { WE } 16 / 20+10 \text { Nitril } \\ \text { 2/polyester }\end{array} & \text { Number of plies } \\ \begin{array}{c}\text { Ǒver : } 1 \mathrm{~mm} \text { nitril } \\ \text { Under : Obelagd }\end{array} & \text { Cover thickness } \\ 160 \mathrm{Kp} & \text { Tensile strength } \\ 1,5 \% & \text { Elongation at } \\ 16 \mathrm{Kp} / \mathrm{cm} \text { Bandbredd } & \text { working load } \\ 3,5 \mathrm{~mm} & \text { Total thickness } \\ 100 \mathrm{~mm} & \text { Min. drumdiameter } \\ -25-+120{ }^{\circ} \mathrm{C} & \text { Working temperature } \\ 1600 \mathrm{~mm} & \text { Max. belt width } \\ \text { Ǒver : } 0,5-0,6 & \text { Friction coefficient } \\ \text { Under : } 0,15-0,2 & \text { Weight } \\ 3,5 \mathrm{~kg} / \mathrm{m}^{2} & \text { Modulus of elasticity } \\ 30 \mathrm{daN} / \mathrm{mm}^{2} & \end{array}$

Fig. 10. - Caractéristiques du nouveau type de courroie de provenance suédoise.

[Physical and mechanical characteristics of possible new charging belt from a Swedish firm.]

est entraînée à la fois par les deux tambours d'extrémité de $\varnothing 315 \mathrm{~mm}$ couplé chacun à un moteur de $37 \mathrm{~kW}$, partageant ainsi la charge de manière à éviter des variations importantes de la tension mécanique locale en régime dynamique (Fig. 11).
Sa trajectoire est imposée, une section morte sur deux, soit tous les $5,64 \mathrm{~m}$, par un minimum de 11 paires de supports (rouleaux ou patins à gaz) positionnés en fonction des flèches statiques et dynamiques sous l'effet du poids propre et des forces 


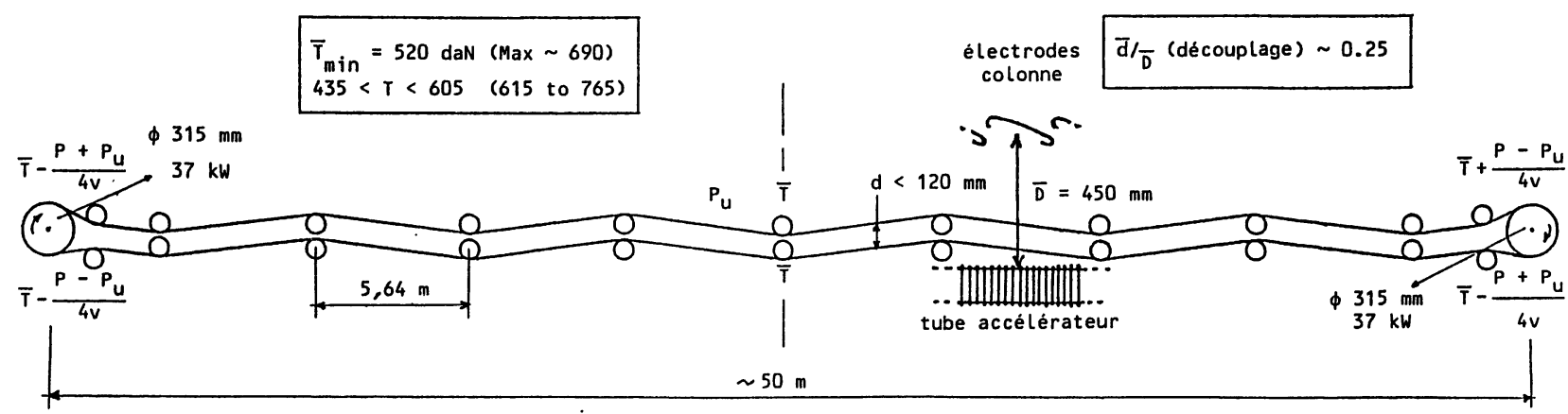

Fig. 11. - Disposition schématique de la courroie pour le Vivitron. La tension mécanique est indiquée en fonction de la tension moyenne $\bar{T}$ au terminal et de la puissance consommée. $P$ est le terme de frottement global (mécanique + électrique), $P_{\mathrm{u}}$ est la puissance électrique disponible au terminal.

[Belt arrangement for the Vivitron. Mechanical belt tension is indicated at different locations as a function of consumed power. $P$ is the electrical and mechanical friction power, $P_{\mathrm{u}}$ is the possible set up power in the terminal electrode where the tension has its mean value $\bar{T}$.]

électriques. Cette disposition a été choisie, en fonction des caractéristiques de la courroie, de manière à assurer un découplage $d / D \sim 0,25$ tout en gardant un fonctionnement correct avec un minimum de supports, quelle que soit la charge.

La tension par brin au repos est de 520 daN (1\% d'allongement) avec laquelle plus de $40 \mathrm{~kW}$ pourraient être transmis.

\section{Stade actuel.}

- La conception de principe est achevée et les principales caractéristiques électrostatiques et mécaniques déterminées.
- Un prototype de patin à gaz a été développé en collaboration avec l'industrie et est prêt pour des essais.

- Un banc d'essai à l'échelle 1 , de $27 \mathrm{~m}$ de longueur (Fig. 12), en court-circuit et à l'air, a été construit et installé dans le but d'étudier le comportement du système et particulièrement l'entraînement partagé par deux moteurs, le fonctionnement dynamique (battements, vibrations), la tenue des rouleaux, le support par coussin de gaz, la génération de puissance électrique au niveau des sections mortes, ainsi que la partie électrostatique, bien qu'à l'air et sans haute tension.

- Les études de réalisation sont en cours. L'ensemble pourrait être prêt fin 1988.

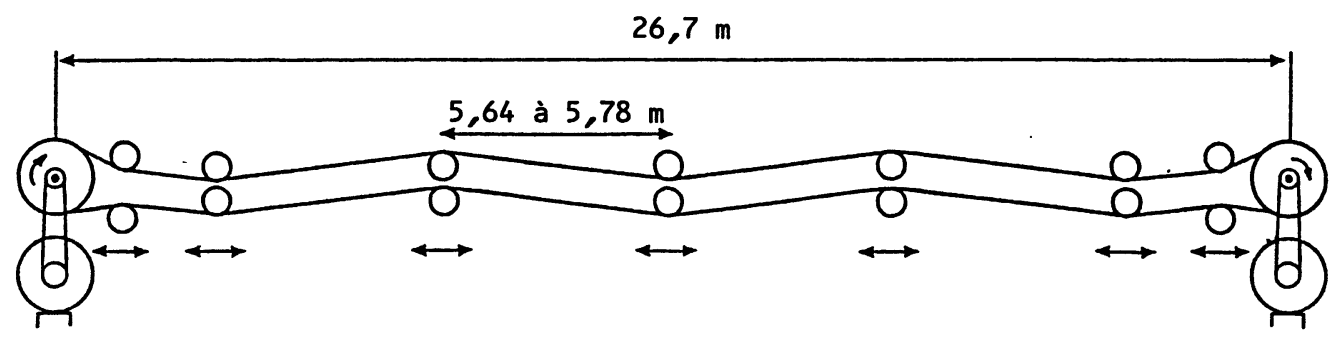

Fig. 12. - Banc d'essai à l'échelle 1 d'une portion du système de charge comportant 4 sections courantes sur 8 au total. Ce banc est à l'air et en court-circuit.

[30 m, one-to-one, charging system test bench.]

Bibliographie

[1] HAAS, F., Applications de techniques nouvelles à la construction de machines électrostatiques. Un exemple : le Vivitron, Commun. au présent congrès.
[2] Helleboid, J. M., Bertein, H., Système de transport de charges électrostatiques de type Van de Graaff pour le Vivitron, RGE 9/85 650-655 et Rapp. interne CRN-VIV-17. 
[3] Helleboid, J. M., Le système de charge du Vivitron, Rapp. interne CRN-VIV-28.

[4] Helleboid, J. M., Belt charging system for the $35 \mathrm{MV}$ Vivitron accelerator, N.I.M. $A 268$ (1988) 414-418 et Rapp. interne CRN-VIV-37.

[5] Hellebroid, J. M., Letournel, M., Essai de courroies de différents types sur l'accélérateur
CN 7 MV， Rapp. d'activité du C.R.N. 1985 p. 162.

[6] Hemryd, B., Rapp. annuel de l'Université d'Uppsala 1984.

[7] ARndT, P., First encouraging experience with a new type of belt material for electrostatic accelerators, Rev. Sci. Instrum. 57 (1986) 735. 